\title{
Multi-objective optimization of nitinol stent design
}

\author{
G. Alaimo ${ }^{\mathrm{a}, *}$, F. Auricchio ${ }^{\mathrm{a}}$, M. Conti ${ }^{\mathrm{a}}$, M. Zingales ${ }^{\mathrm{b}, \mathrm{c}}$ \\ a Dipartimento di Ingegneria Civile e Architettura (DICAR), Università di Pavia, Via Ferrata 3, Pavia 27100, Italy \\ ${ }^{\mathrm{b}}$ Dipartimento di Ingegneria Civile, Ambientale, Aerospaziale e dei materiali (DICAM), Università degli Studi di Palermo, Viale delle Scienze Ed. 8, Palermo \\ 90128, Italy \\ c Bio/NanoMechanics for Medical Sciences Laboratory, ATeN-Center, Viale delle Scienze Ed. 18, Palermo 90128, Italy
}

\section{A R T I C L E I N F O}

\section{Article history:}

Received 16 February 2017

Revised 6 June 2017

Accepted 14 June 2017

Available online $\mathrm{xxx}$

\section{Keywords:}

Fatigue

Multi-objective optimization

Nitinol

Stent

Tapered strut

Structural finite element analysis

\begin{abstract}
A B S T R A C T
Nitinol stents continuously experience loadings due to pulsatile pressure, thus a given stent design should possess an adequate fatigue strength and, at the same time, it should guarantee a sufficient vessel scaffolding. The present study proposes an optimization framework aiming at increasing the fatigue life reducing the maximum strut strain along the structure through a local modification of the strut profile.

The adopted computational framework relies on nonlinear structural finite element analysis combined with a Multi Objective Genetic Algorithm, based on Kriging response surfaces. In particular, such an approach is used to investigate the design optimization of planar stent cell.

The results of the strut profile optimization confirm the key role of a tapered strut design to enhance the stent fatigue strength, suggesting that it is possible to achieve a marked improvement of both the fatigue safety factor and the scaffolding capability simultaneously. The present study underlines the value of advanced engineering tools to optimize the design of medical devices.
\end{abstract}

(c) 2017 IPEM. Published by Elsevier Ltd. All rights reserved.

\section{Introduction}

Nowadays, self-expanding nitinol stents are widely used as part of percutaneous minimally-invasive techniques aimed at treating occluded vessels. Unfortunately, several mechanical failures of such a class of devices have been observed [1]; this drawback often results in loss of scaffolding capabilities of the stent, thrombus formation, and restenosis [2,3]. In particular, partial or total stent fractures have been found in aortic [4], renal [5], and pulmonary [6] implants, as well as in lower limb arteries, i.e., superficial femoral artery (SFA) and popliteal artery [7-10]. Therefore, the long-term structural integrity of a given stent model should be one of the principal design parameter to be taken into account.

Given such considerations, it is necessary to optimize stent design performing a thorough engineering analysis, able to assess the relation between the stent geometry and its structural performance. Such an optimization should increase the fatigue strength without penalizing other biomechanical design requirements, such as the vessel scaffolding.

Despite several studies already addressed the analysis of nitinol stents [11-13] and the literature provides some example of stent design optimization analysis [14-19], to the best of our knowledge,

\footnotetext{
* Corresponding author.

E-mail address: gianluca.alaimo01@universitadipavia.it (G. Alaimo).
}

only two studies deal with fatigue strength enhancement of nitinol stents [20,21]. Abad et al. [20] proposed a planar lattice for the realization of a stent with smooth cell shapes in order to reduce peaks of strain induced by abrupt changes in the stent geometry. The study proposes a single-objective optimization process to minimize the curvature of the elementary unit defining the stent design, i.e., stent cell. Azaouzi et al. [21] presented a single-objective optimization approach based on Kriging response surfaces; such an approach has been used to improve the fatigue strength of the stent by minimizing the strut volume without decreasing the stiffness of the stent. The algorithm considers the strut geometry (width, length, and thickness) as the design variables to be optimized. Both studies combine single-objective optimization methods with structural finite element analysis (FEA).

Moreover, a further literature analysis suggests that: (i) it is possible to enhance fatigue strength acting on the stent cell design but such an improvement has its counterpart in a loss of stiffness [22,23]; (ii) a tapered strut profile enhances the stent fatigue strength, being thus an ideal starting point for the stent design optimization $[24,25]$.

Relying on the previous observations, in the present study we propose a multi-objective optimization procedure acting on both stent cell geometry and strut shape to enhance the fatigue strength of a nitinol stent and its scaffolding capabilities. In particular, the optimization framework accounts for non-linear structural 


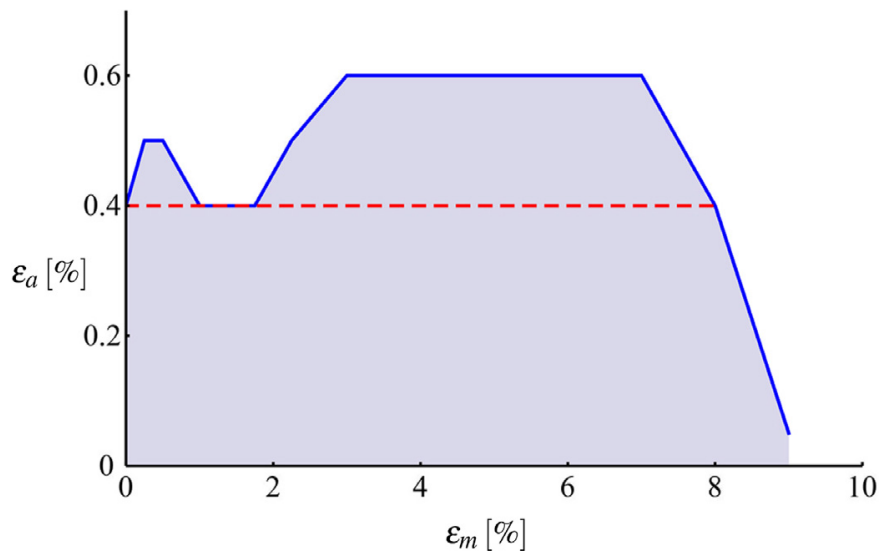

Fig. 1. Constant fatigue life diagram $[23,28]$ : shaded area represents specimens conditions that survived $10^{7}$ cycles while the dashed line represents the value of $\varepsilon_{a}$, i.e., $0.4 \%$, that we adopt in the present paper as conservative threshold.

FEA combined with a Multi Objective Genetic Algorithm (MOGA) [26] based on Kriging response surfaces.

\section{Materials and methods}

\subsection{Fatigue strength analysis}

For the purposes of our study we adopt a strain-based approach, which is the most suitable method to deal with fatigue of nitinol stent, as suggested by Robertson et al. [27]. In particular, in case of time-varying cyclic loads, it is possible to define the mean and the alternating value of the first principal strain, respectively $\varepsilon_{m}$ and $\varepsilon_{a}$, as:

$\varepsilon_{m}=\frac{\varepsilon_{\max }+\varepsilon_{\min }}{2}$

$\varepsilon_{a}=\frac{\left|\varepsilon_{\max }-\varepsilon_{\min }\right|}{2}$

where $\varepsilon_{\max }$ and $\varepsilon_{\min }$ are respectively the maximum and the minimum principal strain values in a loading cycle.

As demonstrated by Pelton etal. [23,28], who tested to failure planar diamond-shape specimens under various combinations of $\varepsilon_{m}$ and $\varepsilon_{a}$, the fatigue strength mainly depends on the alternating value of the first principal strain $\varepsilon_{a}$. As shown by the diagram depicted in Fig. 1, it is possible to define a conservative threshold of $0.4 \%$ for the alternating value of the first principal strain $\varepsilon_{a}$ for any value of mean strain $\varepsilon_{m}$.

It is worth noting that the damage tolerance analysis (DTA) is the alternative method commonly used for the study of fatigue. Such an approach essentially relies on fracture mechanics and Paris-Erdogan law [29]. As concerns nitinol stents, very few studies have been conducted in order to evaluate the fracture strength and other parameters typical of DTA. One reason is that it holds for values of the flaw size larger than a threshold value below which there is no propagation of the fracture: as reported in Robertson and Ritchie [30] this critical flaw size is about $15-50 \mu \mathrm{m}$. Medical devices such as stents have geometric dimensions that are comparable with this threshold value, making DTA useless: it is therefore more useful to focus on prevention rather than on control of the growth of flaws.

For these reasons it can be assumed that the DTA is more appropriate when the typical dimensions of the device are large enough to support the flaw growth or when, for example, the production-process is not sufficiently established to ensure the absence of flaws of critical dimensions [27].

\subsection{Stent geometry}

In order to define the link between the overall size of a typical "v-shaped" stent and the size of the single cell, it is appropriate to refer to the planar projection of the stent obtained from a virtual unrolling, as illustrated in Fig. 2. In this way, the whole stent design can be thought as a repetition of $N$ cells along the circumference ( $y$ axis) and $M$ cells in the axial direction ( $x$ axis). For the optimization procedure, we adopt such a simplified planar model. Accordingly, it is possible to define the following geometrical relation:

$l_{c}=\frac{\pi D}{N}$

where $D$ is the outer diameter of the stent and $l_{c}$ is the length of the unrolled cell in the circumferential direction. Similarly, we have:

$l_{z}=\frac{L}{M}$

where $L$ and $l_{z}$ are the lengths of the whole stent and of the cell in the axial direction, respectively.

A change, $\Delta D$, of the stent diameter $D$ leads to a variation of the cell height, $\Delta l_{c}$, (see Figs. 2 and 3) that, according to Eq. (2), reads:

$\Delta l_{c}=\frac{\pi \Delta D}{N}=2 \delta$

being $\delta$ the displacement, along $y$-direction, experienced by the single strut and due to a given variation of the stent diameter $\Delta D$. Each cell is made up of three basic elements: strut, link, and crown. Two struts and the crown that connects them constitute the v-shape portion of the planar stent cell [31] as shown in Fig. 2. Fatigue strength and scaffolding do not depend only on the cell geometry but also on strut dimensions (width $w$, length $l$, and thickness $t$ ) and on its shape (constant cross-sectional profile versus variable one). Thus, we restrict our attention in relating fatigue strength and scaffolding capabilities to such geometrical quantities: lower will be the alternating value of the first principal strain, higher would be the fatigue strength, while higher will be in-plane cell stiffness (ratio $F / \delta$ as shown in Fig. 3), higher would be stent scaffolding capability.

To this end, let us consider a v-shape portion of the planar stent cell as shown in Fig. 3, subjected to a total displacement $\delta_{\text {tot }}=2 \delta$ in $y$-direction resulting from the application of a load $F$. We consider the strut as a cantilever beam having rectangular cross-section: from simple beam mechanics, under the assumptions of small strain approximation and linear elastic constitutive behavior $[22,23]$, the maximum elongation in the strut is experienced at the outer curvature. The corresponding first principal strain is:

$\varepsilon=Z \frac{w \delta_{\text {tot }}}{l^{2}}$

having the value of $Z=3 / 2$ for rectangular cross-section. Eq. (5) shows that the principal strain $\varepsilon$ is proportional to the strut width $w$ and inversely proportional to the strut length $l$.

We also consider the opening radial force that a nitinol stent applies to the vessel wall after the deployment, namely the chronic outward force (COF) which, in case of "v-shaped" stents, can be evaluated considering the geometry and the mechanics of the single "v-shape" portion $[22,23]$. Stent and vessel interact with radial forces, acting along the $z$-axis in Fig 2, through the external surface of the stent and the internal wall of the vessel. However, forces applied on the vessel wall by a nitinol stent originates from its circumferential stiffness [22,23] and, in the same way, vessel recoil is contrasted by stent internal circumferential forces ( $y$-axis in Fig 2). 


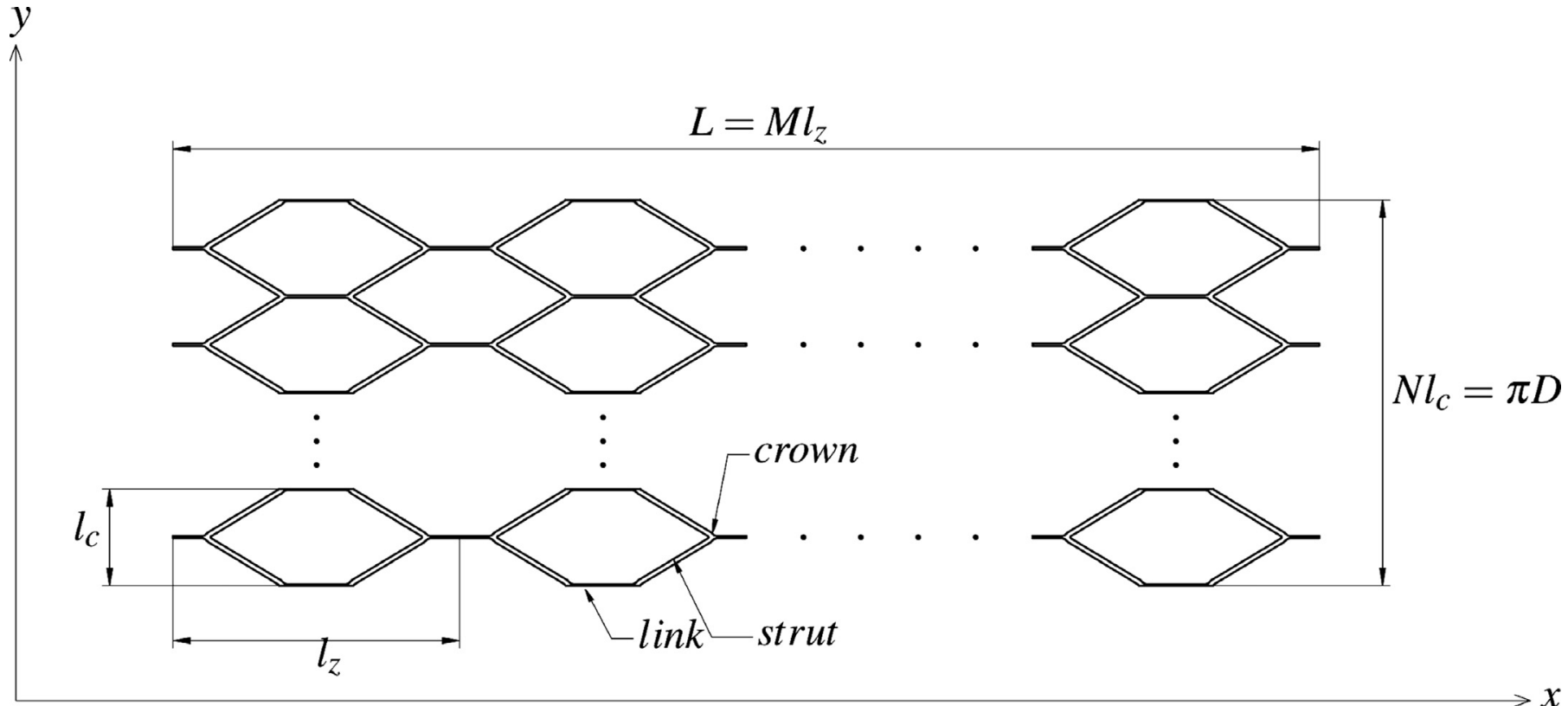

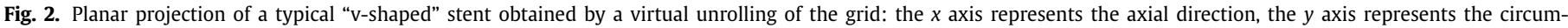
ferential direction while the $z$ axis, orthogonal to the $x y$ plane, is the radial direction. Global dimensions of the stent and the elements of the cell are shown.

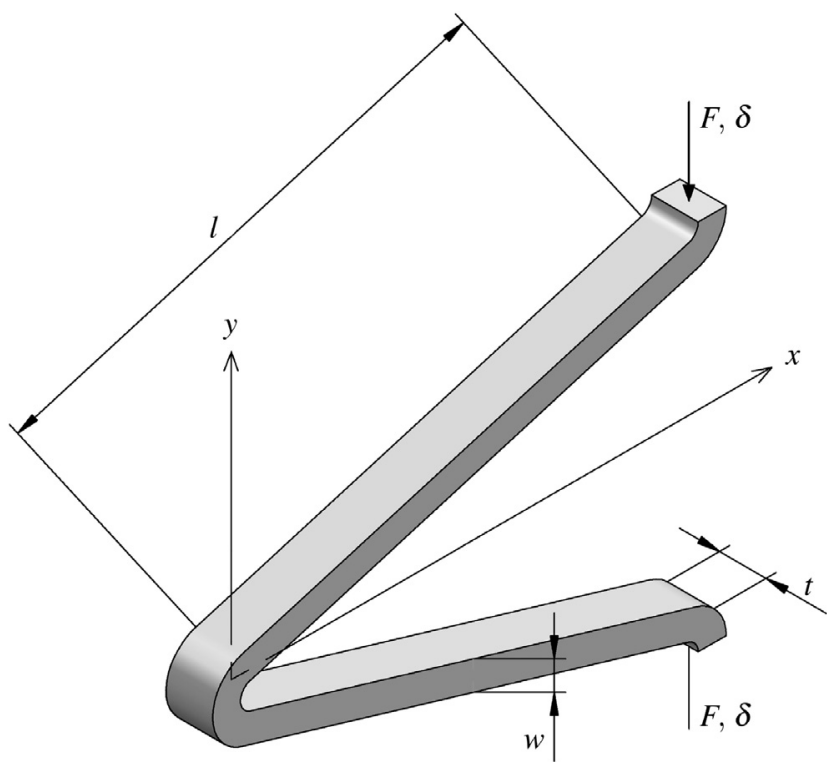

Fig. 3. Stent cell "v-shape" portion with dimensions and applied loads. Axes in $x$ and $y$ directions are parallel to those of Fig. 2.

By analogy, pressure acting on a pipe tend to change its diameter: such changes are opposed by the circumferential stiffness of the pipe. According to such considerations, in the present work, we evaluated COF as the force ( $F$ in Fig 3 ), in the circumferential direction, resulting from a given "v-shape" displacement $\delta$.

A typical nitinol stent force (per unit cell)-diameter curve is reported in Fig. 4, illustrating the concept of biased stiffness. The stent is crimped at diameter of $3 \mathrm{~mm}$ into the delivery system following the loading curve (path $A-B$ ). After the release inside the vessel, the stent expands following the unloading path of the curve (path B-A). When the stent reaches the vessel diameter, i.e. $8.3 \mathrm{~mm}$ (point $\mathrm{C}$ ), it continues to push outward against the vessel wall with the equilibrium force COF equal to $0.3 \mathrm{~N}$. On the other hand, vessel recoil is resisted through the force dictated by the reloading curve (path C-D) which is stiffer than the unloading path: the force generated by the stent to resist radial compression (point $\mathrm{D}$ ) is the ra-

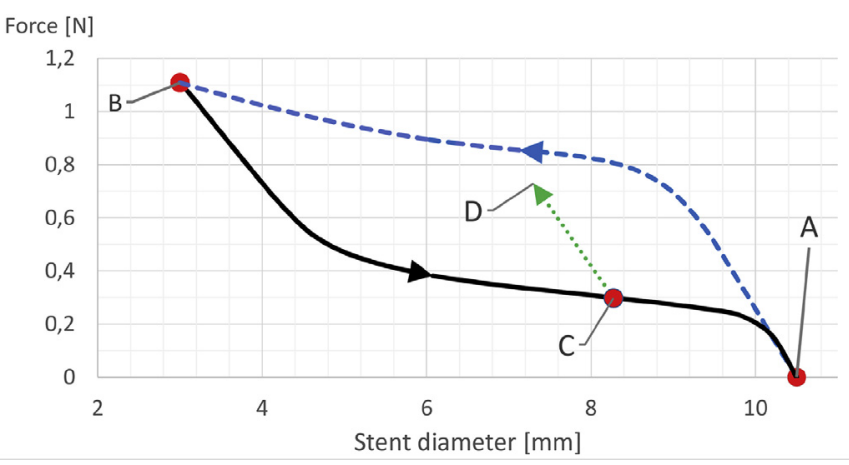

Fig. 4. Force-diameter curve for nitinol stent design $R$. The force is referred to the single cell. Dashed line: loading path $A-B$, continuous line: unloading path B-A, dotted line: reloading path C-D.

dial resistive force (RRF). If the stent is unloaded, it returns to the unloading path, passing through the point $\mathrm{C}$, but with a hysteresis cycle (not shown in figure). From the analysis of Fig. 4 we can conclude that RRF increases rapidly with diameter changes, while COF is nearly constant at $0.3 \mathrm{~N}$ in the neighborhood of point $\mathrm{C}$. Indeed, COF varies from $0.28 \mathrm{~N}$ at a diameter of $8.7 \mathrm{~mm}$ to $0.31 \mathrm{~N}$ at a diameter of $7.9 \mathrm{~mm}$ showing a variation less than $\mp 5 \%$ in this range of diameters. COF is proportional to the cell circumferential stiffness [23] as described by the following equation:

$\mathrm{COF} \propto \frac{t w^{3}}{l^{3}}$

Eq. (6) shows that COF is proportional to $w^{3}$ and inversely proportional to the cube of the strut length $l$. Ideally, we would like to increase COF reducing, at the same time, the maximum principal strain: thus, the considered problem involve two objectives that should be simultaneously optimized. Accordingly, we focus our attention to the class of multi-objective optimization problem.

Remark. The considerations reported in this section are valid only for qualitatively showing the influence of the considered geometrical quantities $(t, w, l)$ on fatigue strength and stiffness of the cell. Indeed, Eqs. (5) and (6) are valid only under the assumption of linear elastic mechanical behavior and small strains approximation. 
Otherwise, the quantity $Z$ in Eq. (5) becomes a function of material and geometry non-linearities. Similar considerations are valid for Eq. (6).

\subsection{Multi-objective optimization}

In classical optimization problems only one objective function is considered. However, real problems often involve several objectives that should be simultaneously optimized. We consider, without loss of generality, a multi-objective minimization problem whose formulation is:

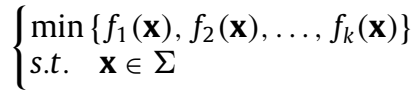

where components $f_{i}: \Sigma \longrightarrow \mathbb{R}, i=1,2, \ldots, k$ are the objective functions and $\Sigma \subset \mathbb{R}^{n}$ is the feasible design domain. Furthermore, we define the objective space $\Omega \subset \mathbb{R}^{k}$ as the image of $\Sigma$, under the mapping function $\mathbf{f}=\left[f_{1}(\mathbf{x}), f_{2}(\mathbf{x}), \ldots, f_{k}(\mathbf{x})\right]$; the elements $\boldsymbol{\omega} \in \Omega$ are the objective vectors. A design vector $\hat{\mathbf{x}}$ and the corresponding solution $\hat{\omega}=\mathbf{f}(\hat{\mathbf{x}})$ of problem (7) are defined trivial if:

$\hat{\omega}_{j}=f_{j}(\hat{\mathbf{x}})=\min _{\mathbf{x} \in \Sigma}\left\{f_{j}(\mathbf{x})\right\}, \quad \forall j=1,2, \ldots, k$

according to definition (8), the design vector $\hat{\mathbf{x}}$ is defined trivial if it minimizes simultaneously all the objective functions $f_{j}, j=$ $1,2, \ldots, k$. Problem ( 7 ) is defined nontrivial if does not exist a trivial solution and, in this case, the objective functions are said to be conflicting. When problem (7) is nontrivial, there exists a (possibly infinite) number of optimal solutions that can be identified introducing the definition of dominance.

A design vector $\hat{\mathbf{x}}_{\mathbf{1}}$ dominates another design vector $\hat{\mathbf{x}}_{\mathbf{2}}$ if both the following conditions are true:

$f_{i}\left(\hat{\mathbf{x}}_{\mathbf{1}}\right) \leq f_{i}\left(\hat{\mathbf{x}}_{\mathbf{2}}\right), \quad \forall i=1,2, \ldots, k$

\section{$\exists j$ such that $f_{j}\left(\hat{\mathbf{x}}_{\mathbf{1}}\right)<f_{j}\left(\hat{\mathbf{x}}_{\mathbf{2}}\right)$}

Eq. (9a) states that the design vector $\hat{\mathbf{x}}_{\mathbf{1}}$ is no worse than $\hat{\mathbf{x}}_{\mathbf{2}}$ for all objectives and Eq. (9b) states that design vector $\hat{\mathbf{x}}_{\mathbf{1}}$ is strictly better than $\hat{\mathbf{x}}_{\mathbf{2}}$ in at least one objective.

Relying on the concept of dominance, the Pareto set is defined as the set of non-dominated solutions of problem (7). From a mathematical point of view, every design in the Pareto set is an equally optimal solution of the multi-objective optimization problem. We conclude noting that we would like to increase COF reducing, at the same time, the maximum principal strain, but the analysis of Eqs. (5) and (6) reveals that such objectives are conflicting. Accordingly, we search for optimal solutions within the Pareto set. The selection of one design from the Pareto optimal set can be done choosing the trade-off point, i.e. the Pareto optimal point that is the most appropriated to the design context [32].

\subsection{Alternative design}

In this section we introduce an alternative design, characterized by the use of a tapered strut, in order to reduce the maximum value of the first principal strain and preserving COF, at the same time. Although it has been observed that the use of tapered profile strut may improve fatigue strength of nitinol stent [25] and such an idea is even covered by patent [24], we note that in the scientific literature, to the best of our knowledge, there are not (i) any specific information about the optimal geometry with respect to the fatigue strength and COF and (ii) available experimental data concerning this type of design.

As an illustrative example we consider the cantilever parabolicprofile, as shown in Fig. 5, whose width $w(x)$ is function of the

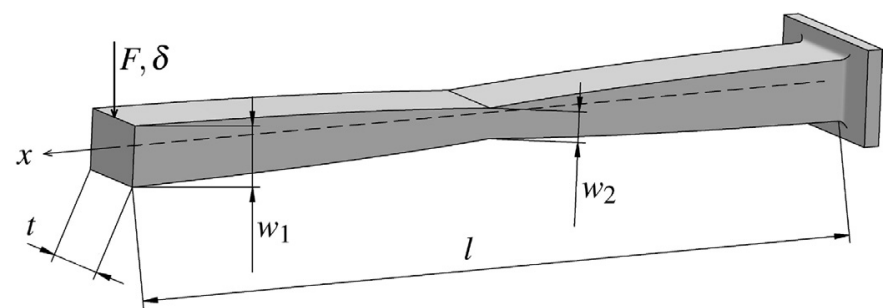

Fig. 5. Cantilever beam with symmetric parabolic profile.

abscissa $x$, as described by the following relation:

$w(x)=w_{1}\left[\sqrt{1-\frac{x}{l}} H\left(\frac{l}{2}-x\right)+\sqrt{\frac{x}{l}} H\left(x-\frac{l}{2}\right)\right]$

where $w_{1}$ is the height of the tips, $l$ the beam length and $H(x)$ is the Heaviside unit step function defined as:

$H(z)=\left\{\begin{array}{lll}1 & \text { if } & z>0 \\ 0 & \text { if } & z \leq 0\end{array}\right.$

The selected parabolic-profile has been chosen to obtain a constant value of the first principal strain (and stress) along the first half of the beam, i.e. for $0<x<l / 2$. Relation (10) shows that $w_{1} / w_{2}=\sqrt{2}$, where $w_{2}=w(l / 2)$ is the width at the center of the beam.

In order to capture the impact of the design on the strut principal strain, we compare its maximum value for the tapered and for the uniform profiles. The comparison is provided assuming the same length $l$ and stiffness $k=F / \delta$ for both solutions, namely $k_{c}$ for uniform profile and $k_{p}$ for the tapered one, being $F$ and $\delta$ the force and the displacement at the free end, respectively.

The analysis is performed relying on the methodology proposed by Romano [33]. Most recent works [34,35] highlight that such an approach must be carefully considered because tapered beams could show a very different mechanical behavior with respect to prismatic ones. However, given the qualitative nature of the present example and considering that we are examining beam exhibiting slow cross-section variations $\left(\left|\frac{d w}{d x}\right|<0.1 \quad \forall x \in[0, l]\right)$, the adopted approach may be considered sufficiently accurate. Summing up, for the constant-section beam of width $w$, the stiffness is:

$k_{c}=\frac{t E w^{3}}{4 l^{3}}$

while, for the parabolic profile, the beam stiffness is:

$k_{p}=\frac{t E w_{1}^{3}}{8 l^{3}}$

where $t$ and $E$ are the thickness and the Young's modulus of the beam, respectively. The same stiffness for both designs can be obtained imposing the equality of Eqs. (13) and (12), yielding $w_{1}=\sqrt[3]{2} w \simeq 1.26 w$.

The expression of the principal strain $\varepsilon_{c}(x)$ for the uniform profile reads:

$\varepsilon_{c}(x)=\frac{3 w \delta}{2 l^{3}}(l-x)$

while, for the parabolic one, we obtain the following relation:

$\varepsilon_{p}(x)= \begin{cases}\frac{3 w \delta}{2 l^{2}} \frac{1}{\sqrt[3]{4}} & \text { if } 0 \leq x \leq \frac{l}{2} \\ \frac{3 w \delta}{2 l^{2}} \frac{1}{\sqrt[3]{4}}\left(\frac{l}{x}-1\right) & \text { if } \frac{l}{2}<x \leq l\end{cases}$

The expressions of the normalized principal strain along the nondimensional coordinate $x / l$ are shown in Fig. 6. Both solutions have 


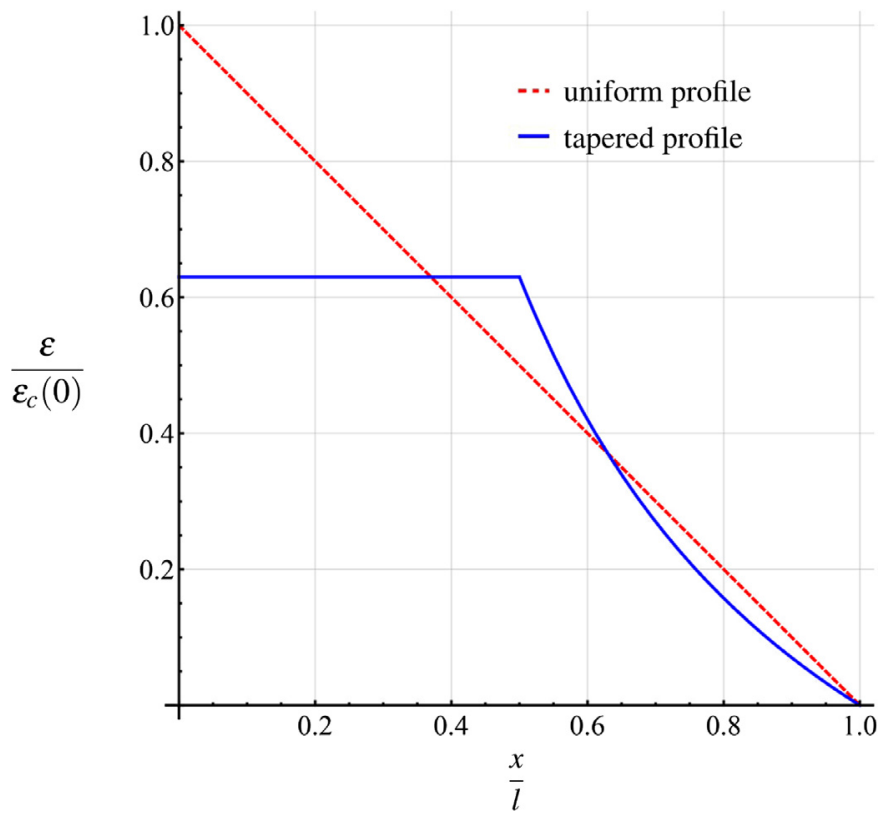

Fig. 6. Graph of the deformation along the $x$ axis normalized with respect to the maximum that occurs in the constant-section beam $\varepsilon_{c}(0)$.

their maximum for $x=0$ and their ratio is:

$\frac{\varepsilon_{p}(0)}{\varepsilon_{c}(0)}=\frac{1}{\sqrt[3]{4}} \simeq 0.63$

Eq. (16) shows that, keeping the same stiffness, length $l$, and thickness $t$, the considered parabolic-profile beam (10) has a maximum value of strain which is substantially lower than the one experienced by the constant-section beam. Additionally, Fig. 6 shows that, in case of constant section, the principal strain $\varepsilon_{c}$ varies linearly along the beam axis while the parabolic profile (10) exhibits a constant distribution of the first principal strain along the first half of the beam and then ramps down to zero with a hyperbolic law. Clearly, when considering profiles different to (10), strain distribution along the beam $x$-axis will be different but, from a qualitative point of view, well-designed tapered profiles contribute to uniform strain field and exhibit lower values of the maximum first principal strain, compared to prismatic ones. Consequently, we conclude that acting on geometrical quantities such as $l, w_{1}, w_{2}$, it is possible to define enhanced geometries that may provide longterm fatigue strength when considered in a structural optimization context.

Remark. Parabolic profile (10) only depends on two independent parameters: we choose the width $w$ and the length $l$ of the beam. Accordingly, the corresponding beam stiffness (13) does not depend on $w_{2}$. The ratio $w_{1} / w_{2}$ can be varied only changing the functional relationship (10).

\subsection{Finite element model}

The considerations reported in Sections 2.1 and 2.4 are used to generate a virtual model of the stent-cell to be used in the structural FEA. Numerical simulations are performed using the commercial software Ansys.

The optimization of the stent cell is obtained in terms of control variables corresponding to the geometrical features of the tapered profile, collected in the vector $\mathbf{x}$ defined as (see Fig. 7):

$\mathbf{x}=\left[w_{1}, l, a, b, c\right]^{T}$ where $l$ is the length of the strut, $w_{1}$ the width at the ends of the strut, and $a, b$ and $c$ dimensionless variables defined as:

$a=\frac{w_{2}}{w_{1}}$

$b=\frac{r}{w_{1}}$

$c=\frac{w_{k}}{w_{1}}$

being $w_{2}$ the width at the midpoint of the strut, $r$ the crown outer radius and $w_{k}$ the width of the link. Nitinol stents are usually lasercut from a standard tube using a crimped design and then expanded and heat-treated to reach the final diameter. In crimped design, the thickness of the strut is usually constant for the convenience of the laser-cutting process and the saving of expensive materials. If the thickness is changed, the diameter of the compact design has to be also changed, thus the original tube will not be compatible. For the mentioned reason, during the optimization process, the thickness $t$ is kept fixed, i.e. $t=200 \mu \mathrm{m}$.

The overall cell dimensions $l_{c}$ and $l_{z}$ are assumed constant during the optimization procedure because they depend on the number of cells in the circumferential and the longitudinal directions, namely $N$ and $M$, as defined by Eqs. (2) and (3) respectively. Indeed, variations of these dimensions may result in an excessive variation of the cell surface that is an important feature for the stent performance.

Cell geometry is also defined by the angle $\phi$ and the length of the link $l_{k}$ (see Fig. 7) that are related to the chosen values of $l_{c}$ and $l_{z}$ and to the input variables (Eq. (17)) as:

$l_{c}=2 l \cos \phi+2(2 b-1) w_{1} \sin \phi$

$l_{z}=2 l_{k}+2 l \sin \phi+4\left[b w_{1}-w_{1}\left(b-\frac{1}{2}\right) \cos \phi\right]$

that resolved with respect to $\phi$ and $l_{k}$ yield:

$\phi=\arctan \left[\frac{l\left(l_{c} w_{1}(2 b-1)+A\right)}{l^{2} l_{c}+(1-2 b) w_{1} A}\right]$

$l_{k}=\frac{l_{z}}{2}-l \sin \phi-2\left[b w_{1}-w_{1}\left(b-\frac{1}{2}\right) \cos \phi\right]$

where $A=l\left(4 l^{2}-l_{c}^{2}+4(1-2 b)^{2} w_{1}^{2}\right)^{1 / 2}$. From Eqs. (20a) and (20b), it can be observed that the parameters $a$ and $c$ do not affect the overall geometry of the cell, but they only have a local effect, in particular on widths $w_{2}$ and $w_{k}$, respectively. The strut profile is represented by a spline curve.

Nitinol super-elastic behavior is simulated by the Shape Memory Alloy (SMA) model available in Ansys [36]; the SMA characteristics have been assumed coincident with those reported by Meoli etal. [37] and shown in Fig. 8. More in detail, $\sigma_{s}^{A S}$ and $\sigma_{f}^{A S}$ are the values of stress at the start and at the end of the austenite-martensite (AM) transformation, $\sigma_{S}^{S A}$ and $\sigma_{f}^{S A}$ are the initial and final stress in the martensite-austenite (MA) transformation, $E_{a}$ and $E_{m}$ are the moduli of elasticity of austenite and martensite, $v$ is the Poisson's ratio and $\varepsilon_{L}$ is the maximum equivalent strain at the end of the AM transformation. The parameter $\alpha$ takes into account the different behavior in tension and compression and it is defined by the following equation:

$\alpha=\frac{\left(\sigma_{s}^{A S}\right)_{c}-\left(\sigma_{s}^{A S}\right)_{t}}{\left(\sigma_{s}^{A S}\right)_{c}+\left(\sigma_{s}^{A S}\right)_{t}}$ 


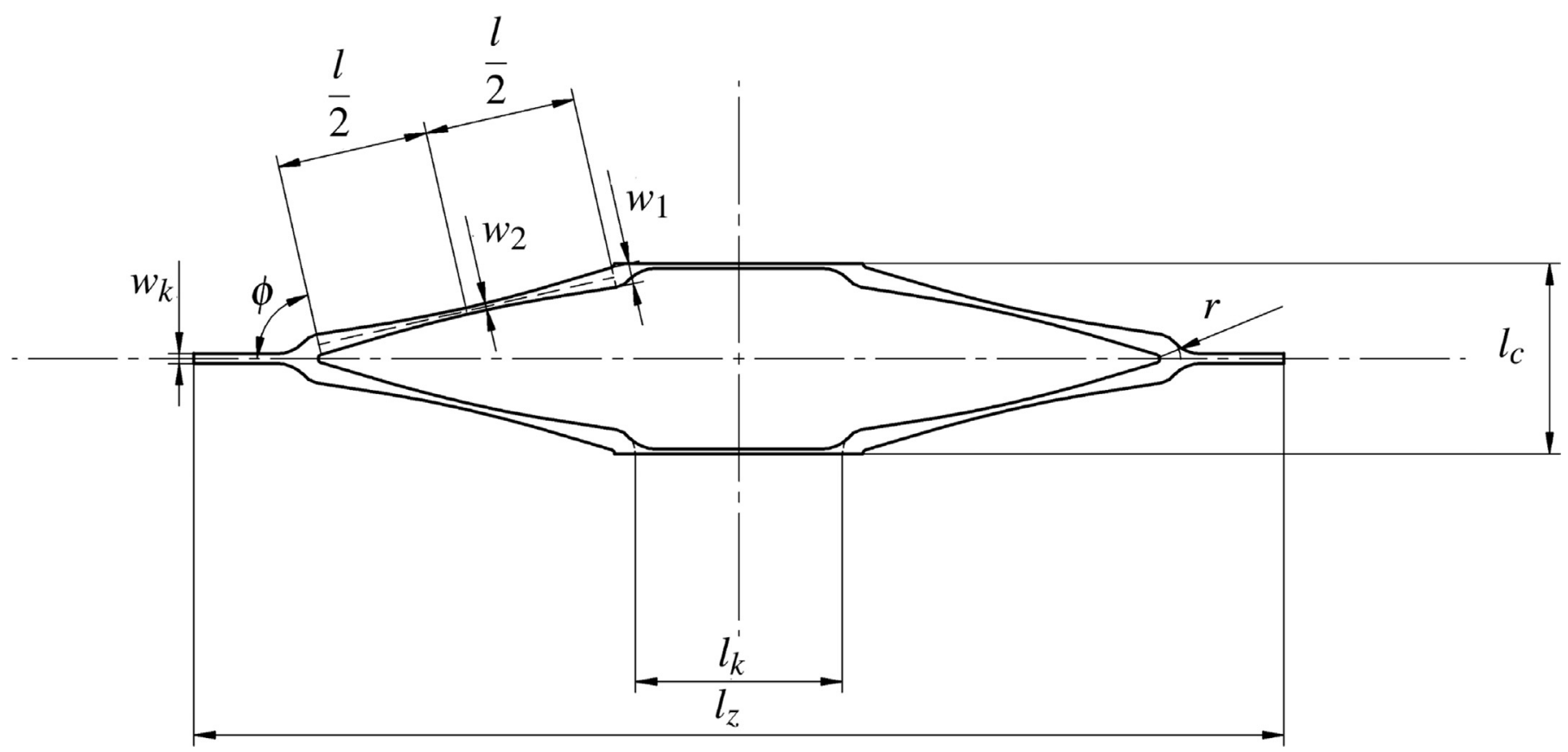

Fig. 7. Parametric model of the stent-cell.

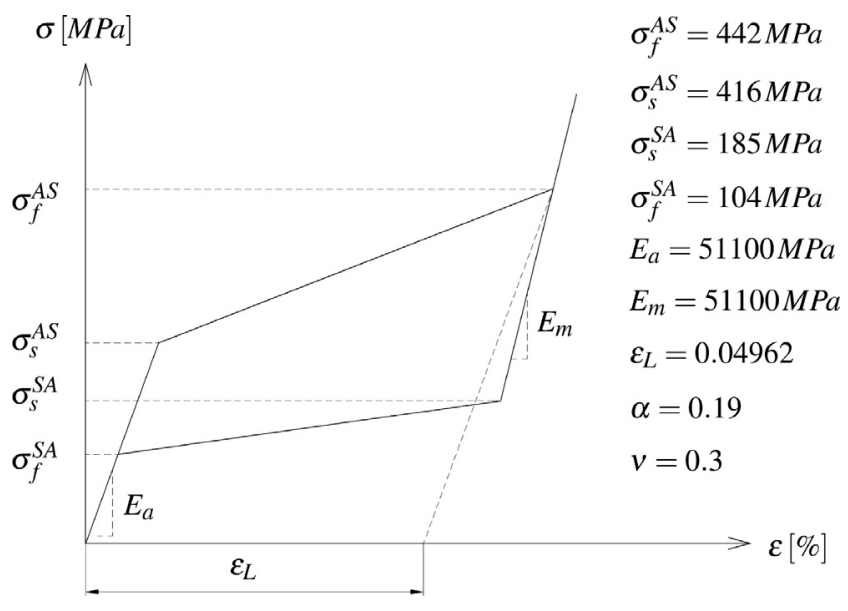

Fig. 8. Curve $\sigma-\varepsilon$ for an ideal super-elastic material.

where $\left(\sigma_{s}^{A S}\right)$ and $\left(\sigma_{s}^{A S}\right)$, are respectively the compressive and tensile stress at the start of the AM transformation. The cell model is discretized with the 8-node brick element Solid185 available in Ansys element library, with full integration. A convergence analysis was performed to ensure a suitable mesh refinement. The quantity selected during the convergence analysis was the maximum value of the first principal strain on the whole strut, after the crimping inside the delivery system (end of step 1 ). The analysis was performed decreasing the average element size in step of $1 \mu \mathrm{m}$ in the range $20-10 \mu \mathrm{m}$ and using several geometries of the strut. The best trade-off between solution accuracy and efficiency was obtained using an element size of $12 \mu \mathrm{m}$. The adopted mesh involves a number of elements which varies, approximately, between 30000 and 40000 according to the considered design.

Fig. 9a shows the whole cell and its geometrical planes of symmetry $\alpha, \beta$ and $\gamma$. We simulated only the strut imposing symmetry boundary conditions on the surfaces resulting from the intersection of the cell with such planes. In particular (see Fig. 9b), on the surface $F$ parallel to the $x y$ plane, displacements $\delta$ along $z$, due to the changes in diameter $D$, are imposed. On the surface $A \subset \beta$ parallel to the $x y$ plane, displacements in the $z$ direction are pre- vented, on the surface $B \subset \gamma$ parallel to the $x z$ plane, displacements in the $y$ direction are prevented, while on the surface $C \subset \alpha$ parallel to the $y z$ plane, displacements in the $x$ direction are prevented.

The stent under investigation is characterized by a diameter $D=10.5 \mathrm{~mm}$ and a length $L=42 \mathrm{~mm}$; then, considering a number of cells equal to $N=14$ and $M=7$ in the circumferential and axial directions respectively, by the relations (2) and (3) we obtain the circumferential length $l_{c}$ and the axial length $l_{z}$ of the cell as $l_{c}=2.4 \mathrm{~mm}$ and $l_{z}=7 \mathrm{~mm}$. The considered loading history (Fig. 10) involves four distinct steps:

Step 1. Stent crimping: the stent diameter goes from diameter $D_{0}=10.5 \mathrm{~mm}$ to diameter $D_{1}=3 \mathrm{~mm}$. It follows (Eq. (4)) that the displacement in the $z$ direction of the surface $F$ (Fig. 9b) reads $\delta_{1}=841 \mu \mathrm{m}$.

Step 2. Stent deployment into the vessel (femoral artery): the stent diameter goes from diameter $D_{1}=3 \mathrm{~mm}$ to diameter $D_{2}=D_{s}=9.6 \mathrm{~mm}$ which coincides with the diameter of the stent at the end of the systolic phase [23]. The resulting displacement, in agreement with the Eq. (4), is equal to $\delta_{2}=101 \mu \mathrm{m}$.

Step 3. Contraction of the stent due to the diastolic blood pressure: the stent diameter goes from $D_{2}=9.6 \mathrm{~mm}$ to diameter $D_{3}=D_{d}=9.3 \mathrm{~mm}$ coincident with the diameter at the end of the diastolic phase; the displacement at the end of this step is equal to $\delta_{3}=135 \mu \mathrm{m}$.

Step 4. Expansion of the stent: return to systolic diameter $D_{4}=D_{s}=9.6 \mathrm{~mm}$, corresponding to the cycle end.

In order to evaluate the change in diameter $\Delta D=D_{s}-D_{d}$ in steps 3 and 4 , resulting from a pressure variation $\Delta p$, we set the arterial compliance to be (after [38]):

$C=100 \frac{D_{s}-D_{d}}{D_{d}}=3.26 \%$

corresponding to a variation in the blood pressure $\Delta p=100 \mathrm{mmHg}$; similar compliance values are obtained in [39]. According to [38], the corresponding cross sectional arterial stiffness $K_{A}$, i.e. the inverse of the compliance per unit of length of 


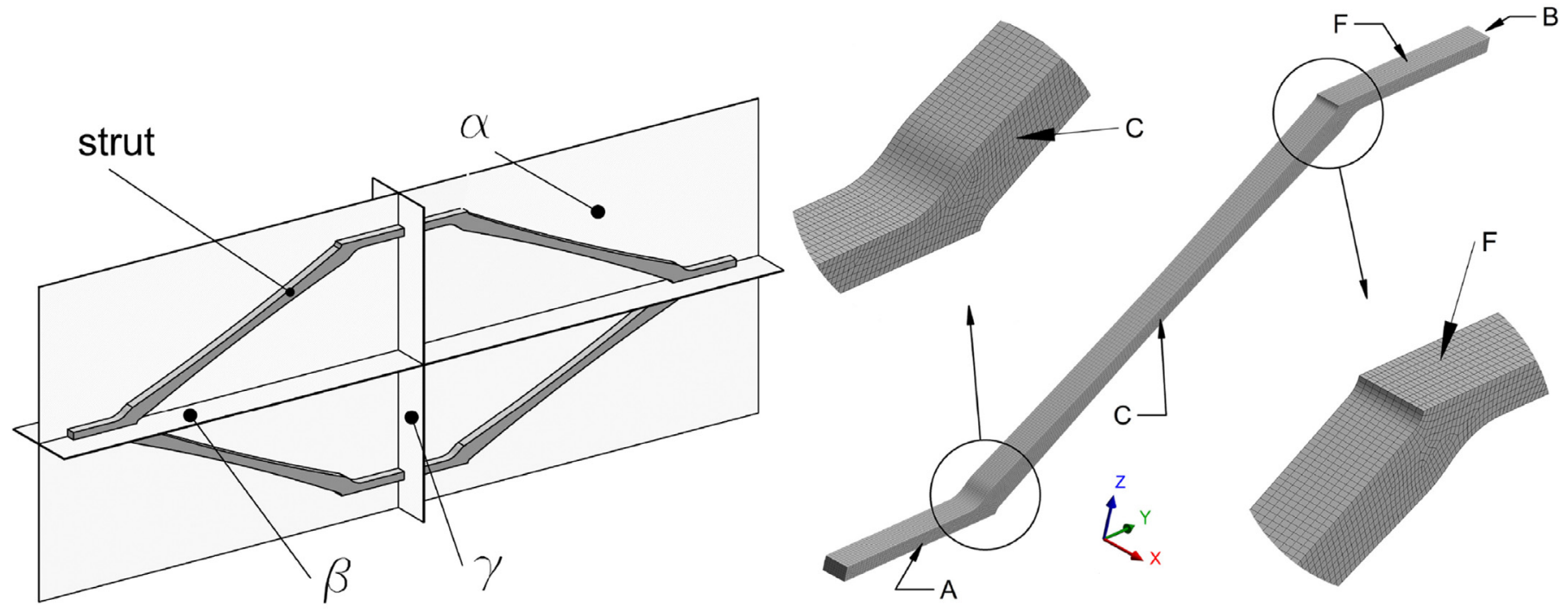

(a)

(b)

Fig. 9. Finite element model. (a) Planes of symmetry $\alpha, \beta$ and $\gamma$ for the unit cell. (b) FE-model with boundary conditions and some details of the mesh.

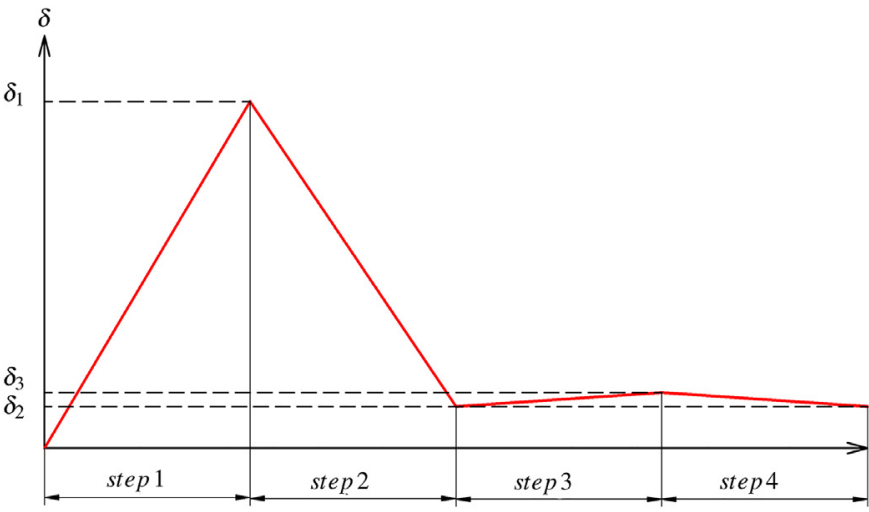

Fig. 10. Displacement history imposed on strut free end. Step 1: stent crimping, step 2: stent deployment, step 3, step 4: stent contraction and expansion, respectively, due to blood pressure variation.

the vessel is:

$K_{A}=\frac{d p}{d A}=\left(\frac{1}{l} \frac{d V}{d p}\right)^{-1}=12.1 \frac{\mathrm{mmHg}}{\mathrm{mm}^{2}}$

where $A, l$ and $V$ are the cross sectional area, the length and the volume of the vessel, respectively.

The quantities of interest are the alternating maximum value of the first principal strain $\varepsilon_{a}$ and the chronic outward force COF. In order to evaluate them, we coded an Ansys Parametric Design Language (APDL) script. More in detail, at the end of steps 3 and 4 for each element of the model the values of the first principal strain are evaluated, and then the mean values $\varepsilon_{m}$ and the alternating value $\varepsilon_{a}$ are calculated: the maximum value of $\varepsilon_{a}$ is stored for each design point $\mathbf{x}$. Similarly, a quantity proportional to COF, represented by the force acting on the surface $F$ (indicated in Fig. 9b) at the end of step 2 and necessary to keep the displacement $\delta_{2}$, is computed and stored.

Remark. As observed in Section 2.2, COF is the opening force that the nitinol stent applies to the vessel wall after the deployment. It can be indifferently evaluated at the end of step 2 or step 4 , but not at the end of step 3 , because of the biased stiffness of nitinol stents.

\subsection{Kriging response surfaces}

For completeness, a brief description of Kriging surfaces (KS) [40-42] is given. KS predict the response $z(\mathbf{x})$ of a function $f(\mathbf{x})$ at unobserved design points $\mathbf{x} \in \Sigma \subset \mathbb{R}^{n}$ relying on the known values $f\left(\tilde{\mathbf{x}}^{i}\right)$ at all the sampled points $\tilde{\mathbf{x}}^{i}, i=1,2, \ldots, M$.

We define $\tilde{\mathbf{f}}$ as the vector of dimension $M$, that contains the values of the function $f(\mathbf{x})$ at each sampled point, namely $\tilde{\mathbf{f}}=\left[f\left(\tilde{\mathbf{x}}^{1}\right), f\left(\tilde{\mathbf{x}}^{2}\right), \ldots, f\left(\tilde{\mathbf{x}}^{M}\right)\right]^{T}$. The general expression of KS is:

$z(\mathbf{x})=d(\mathbf{x})+r(\mathbf{x})$

where $d(\mathbf{x})$ is a polynomial function of $\mathbf{x}$, and $r(\mathbf{x})$ is the realization of a normally distributed Gaussian random process with zero mean, variance $\sigma_{r}^{2}$ and non-zero covariance. The term $d(\mathbf{x})$ in Eq. (24) is usually termed as "trend" as it globally approximates the unknown function $z(\mathbf{x})$ over the feasible design space $\Sigma$, while the term $r(\mathbf{x})$ allows for local deviations, enabling KS to interpolate the $M$ sampled points. The term $d(\mathbf{x})$ is defined as:

$d(\mathbf{x})=\mathbf{q}^{T}(\mathbf{x}) \mathbf{v}$

where $\mathbf{q}(\mathbf{x})=\left[q_{1}(\mathbf{x}), q_{2}(\mathbf{x}), \ldots, q_{m}(\mathbf{x})\right]^{T}$ is the polynomial basis vector, which dimension $m$ depends on the degree of $d(\mathbf{x})-2$ in the present work- and on the dimension $n$ of the design space $\Sigma ; \mathbf{v}=\left[v_{1}, v_{2}, \ldots, v_{m}\right]^{T}$ is a vector of unknown coefficients. The covariance matrix $\mathbf{C}$ of $r(\mathbf{x})$ is a $M \times M$ matrix, whose elements are given by:

$C_{i j}=\sigma_{r}^{2} R_{i j}$

where $R_{i j}$ are the elements of $\mathbf{R}$ representing the spatial correlation functions between each pair $\left(\tilde{\mathbf{x}}^{i}, \tilde{\mathbf{x}}^{j}\right)$ of the sampled points. The spatial correlation function used in the present work is a Gaussian correlation function defined as:

$R_{i j}=R\left(\tilde{\mathbf{x}}^{i}, \tilde{\mathbf{x}}^{j}\right)=\exp \left(-\sum_{k=1}^{n} \lambda_{k}\left|\tilde{x}_{k}^{i}-\tilde{x}_{k}^{j}\right|^{2}\right)$

where $\lambda_{k}, k=1,2, \ldots, n$, are unknown correlation parameters and the quantities $\tilde{x}_{k}^{i}$ and $\tilde{x}_{k}^{j}$ are the $k^{\text {th }}$ components of the sampled points $\tilde{\mathbf{x}}^{i}$ and $\tilde{\mathbf{x}}^{j}$, respectively. Given such assumptions, the vector of unknown coefficients $\mathbf{v}$ in Eq. (25) is obtained by least square 


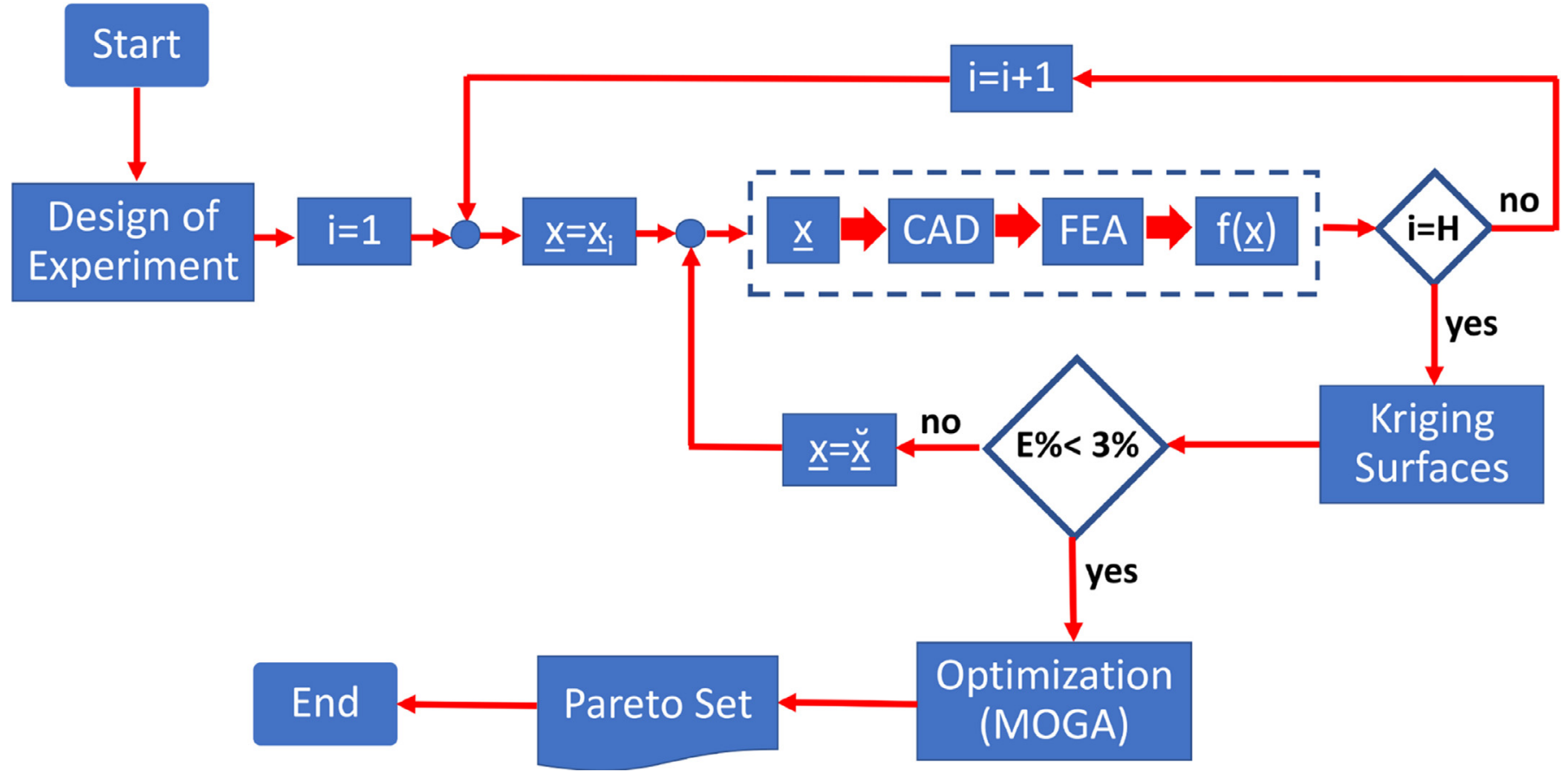

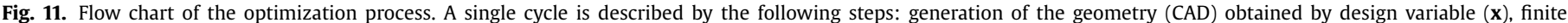

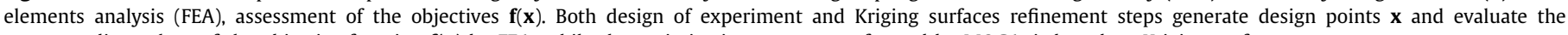
corresponding values of the objective function $\mathbf{f}(\mathbf{x})$ by FEA, while the optimization process, performed by MOGA, is based on Kriging surfaces.

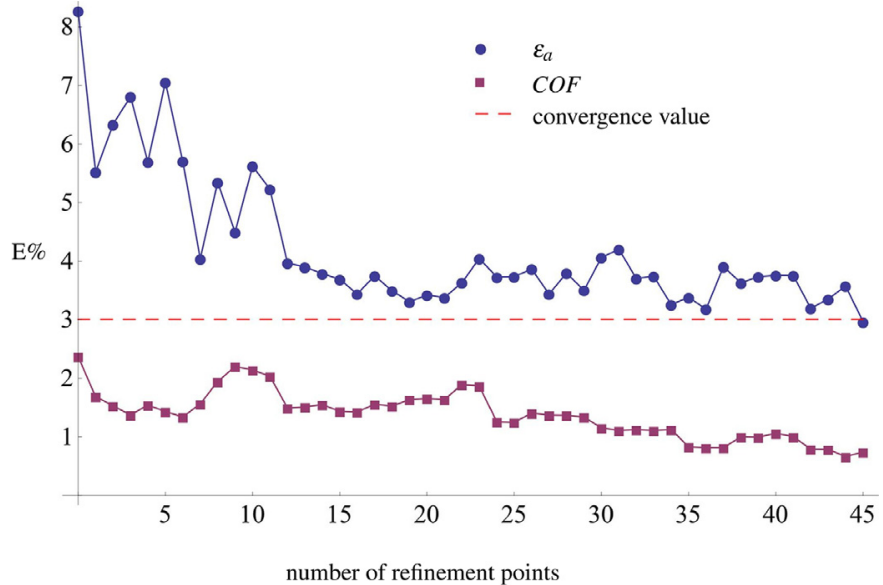

Fig. 12. Graph of the maximum expected relative error $E \%$ versus number of refinement points relative to the Kriging surfaces. It is shown that 45 additional refinement points were needed to reach the selected value of $3 \%$ for the maximum predicted relative error. Red dashed line represents the convergence value. (For interpretation of the references to colour in this figure legend, the reader is referred to the web version of this article.)

regression, obtaining:

$\tilde{\mathbf{v}}=\left(\mathbf{Q}^{T} \mathbf{R}^{-1} \mathbf{Q}\right)^{-1} \mathbf{Q}^{T} \mathbf{R}^{-1} \tilde{\mathbf{f}}$

where $\mathbf{Q}$ is the $M \times m$ matrix defined as:

$\mathbf{Q}=\left[\begin{array}{c}\mathbf{q}^{T}\left(\tilde{\mathbf{x}}^{1}\right) \\ \mathbf{q}^{T}\left(\tilde{\mathbf{x}}^{2}\right) \\ \vdots \\ \mathbf{q}^{T}\left(\tilde{\mathbf{x}}^{M}\right)\end{array}\right]$

KS in Eq. (24) is thus obtained as:

$z(\mathbf{x})=\mathbf{q}^{T}(\mathbf{x}) \tilde{\mathbf{v}}+\mathbf{g}^{T}(\mathbf{x}) \mathbf{R}^{-1}(\tilde{\mathbf{f}}-\mathbf{Q} \tilde{\mathbf{v}})$ where $\mathbf{g}(\mathbf{x})$ is the correlation vector between the point $\mathbf{x}$ and the sampled data points, namely:

$\mathbf{g}(\mathbf{x})=\left[R\left(\mathbf{x}, \tilde{\mathbf{x}}^{1}\right), R\left(\mathbf{x}, \tilde{\mathbf{x}}^{2}\right), \ldots, R\left(\mathbf{x}, \tilde{\mathbf{x}}^{M}\right)\right]^{T}$

Correlation parameters $\lambda_{k}$ in Eq. (27) are to be determined before the KS can be computed. They are evaluated maximizing, over $\lambda_{k}$, $k=1,2, \ldots, n$, the following functional $\Phi$ :

$\Phi\left(\lambda_{1}, \lambda_{2}, \ldots, \lambda_{n}\right)=M \ln \left(\sigma^{2}\right)+\ln (\operatorname{det} \mathbf{R})$

where $\sigma^{2}$ is the estimate of variance $\sigma_{r}^{2}$ in Eq. (26), that is given by:

$\sigma^{2}=\frac{(\tilde{\mathbf{f}}-\mathbf{Q} \tilde{\mathbf{v}}) \mathbf{R}^{-1}(\tilde{\mathbf{f}}-\mathbf{Q} \tilde{\mathbf{v}})}{M}$

The predicted deviation of the KS from the actual response, for each point $\mathbf{x} \in \Sigma$, is statistically represented by the root mean squared error $E(\mathbf{x})$, that is defined as:

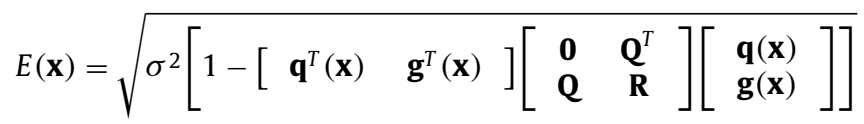

Typical steps in defining KS are: the evaluation of correlation parameters $\lambda_{k}$ by maximizing non-linear functional (32), determination of the correlation matrix $\mathbf{R}$ by (27), evaluation of $\mathrm{KS}$ (30) through (28) and (31), error estimation (34).

\subsection{Optimization}

We assume that the domain $\Sigma \subset \mathbb{R}^{5}$ of the multi-objective optimization is bounded within the following intervals:

$w_{1} \in[80,200]$

$l \in[1500,2500]$ 


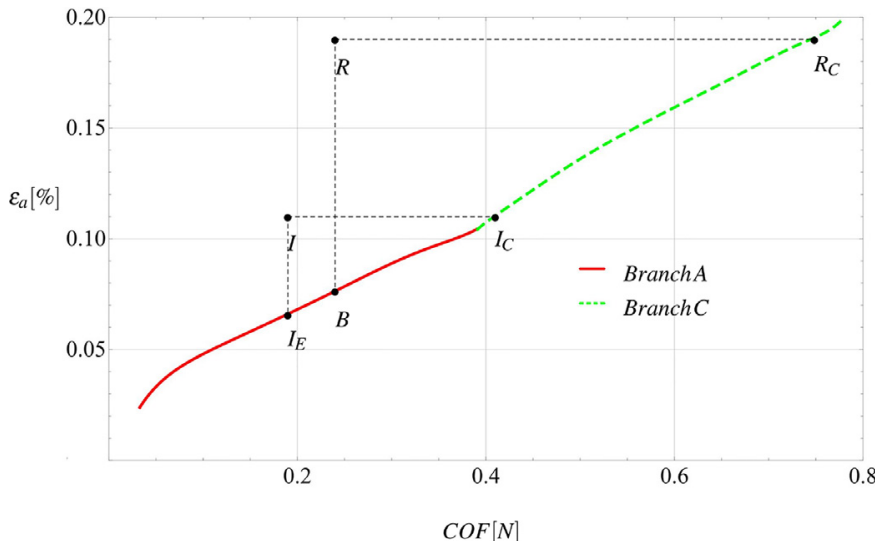

Fig. 13. Set of Pareto optimal-points. Two main different zone are present: branch $A$ and branch $C$. Point $R$ represents the constant-section strut design, point $B$ represents the optimized tapered strut and point $I$ represents the same geometry of the design $R$ but with tapered profile.

$a \in[0.35,1]$

$b \in[1.2,1.5]$

$c \in[0.5,1.5]$

where $w_{1}$ and $l$ are expressed in $\mu \mathrm{m}$ and $a, b$ and $c$ are dimensionless. The range of variation of the input variables, has been chosen considering the typical dimensions of the strut composing commercial nitinol stents. In particular, we chose as reference design $\mathbf{x}_{R}$ the Smart stent by Cordis [43] (see [23,37]), having a constant cross section strut $(a=1)$ with length $l$, height $w_{1}$ and thickness $t$ equal to $2000 \mu \mathrm{m}, 120 \mu \mathrm{m}$ and $200 \mu \mathrm{m}$, respectively; other parameters $b$ and $c$ are equal to 1.25 and 1 respectively. Accordingly, the reference design $\mathbf{x}_{R}$ is obtained with our parametric model, employing the following design variables:

$\mathbf{x}_{R}=\left[w_{1}, l, a, b, c\right]^{T}=[120,2000,1,1.25,1]^{T}$

The array of the objective functions is defined as:

$\mathbf{f}(\mathbf{x})=\left[\varepsilon_{a}(\mathbf{x}), \operatorname{COF}(\mathbf{x})\right]^{T}$

We preliminarily run a FEA to simulate the reference design $\mathbf{x}_{R}$, obtaining an alternating strain $\varepsilon_{a}$ equal to $0.19 \%$ with $\mathrm{COF}=0.24 \mathrm{~N}$ (point $R$ in Fig. 13). Assuming a fatigue limit of $\varepsilon_{L}=0.4 \%$ as discussed in Section 2.1, for the reference design the safety factor yields:

$S_{R}=\frac{\varepsilon_{L}}{\varepsilon_{a}}=\frac{0.4}{0.19}=2.1$

The multi-objective optimization aims at minimizing $\varepsilon_{a}(\mathbf{x})$ and, at the same time, maximizing $\operatorname{COF}(\mathbf{x})$, with $\mathbf{x} \in \Sigma$. Furthermore, the additional constraint $\varepsilon_{a} \leq 0.2 \%$ is imposed to admit only $\mathbf{x}$ (i.e. designs) corresponding to a value of the fatigue safety factor $S \geq 2$ : in this way we consider only solutions having safety factor comparable with or higher than the reference design $\mathbf{x}_{R}$. The mathematical formulation of the considered optimization problem reads:

Objectives: $\left\{\begin{array}{l}\min \left\{\varepsilon_{a}(\mathbf{x})\right\} \\ \max \{\operatorname{COF}(\mathbf{x})\}\end{array}\right.$

Constraints: $\left\{\begin{array}{l}\varepsilon_{a}(\mathbf{x}) \leq 0.2 \\ \mathbf{x} \in \Sigma\end{array}\right.$
The goal of the multi-objective optimization is to evaluate the Pareto set: to obtain it we use a MOGA $[26,44]$ based on KS. In the present work we use KS for each component of the objective vector $\mathbf{f}(\mathbf{x})$. KS are preliminarily built during the domain mapping phase (see Fig. 11), which is essentially the selection of a set of points $\mathbf{x}$ in which $\mathbf{f}(\mathbf{x})$ is evaluated by FEA. Such initial set is obtained with the Central Composite Design (CCD) method [44]. According to this approach, the objective vector $\mathbf{f}(\mathbf{x})$ is evaluated for $H$ design points, with $H=2^{m}+2 m+n_{c}=54$ where $m$ is the number of input variables $(m=5)$, and $n_{c}=12$ is the number of auxiliary points that allow for estimation of second-order effects.

Once the initial mapping of $\mathbf{f}(\mathbf{x})$ is completed (design of experiment step in Fig. 11), a first KS interpolation of the sampled points is performed, for each objective function. The greater is the number of points used to construct the surface, the lower will be its deviation from the exact value. An error estimator of KS is obtained introducing the maximum predicted relative error $E_{i} \%$, defined as:

$E_{i} \%=\frac{100}{f_{i}^{\max }-f_{i}^{\min }} \max _{\mathbf{x} \in \Sigma}\left[E_{i}(\mathbf{x})\right]=\frac{100}{f_{i}^{\max }-f_{i}^{\min }} E_{i}\left(\breve{\mathbf{x}}_{i}\right), \quad i=\varepsilon_{a}, \mathrm{COF}$

where $f_{i}^{\max }$ and $f_{i}^{\min }$ are the maximum and the minimum known values (on design points $\mathbf{x}$ ) of the objective $f_{i}$ under study, respectively, $E_{i}(\mathbf{x})$ is the root mean square error defined in Eq. (34) and $\breve{\mathbf{x}}_{i}$ is the design point for which the error $E_{i}(\mathbf{x})$ is maximum. Clearly, the error $E_{i} \%$ is not the same for both the objective functions: we define the error $E \%=\max _{i}\left[E_{i} \%\right]$ and $\breve{\mathbf{x}}$ the point in which $E \%$ occurs. We opt for a value of the maximum predicted relative error of $3 \%$ as best trade-off between computational time needed for solving KS and accuracy. In order to obtain the requested value of $E \%$, for each response surface refinement step, we generate the additional refinement points $\breve{\mathbf{x}}$, and we use the corresponding $\mathbf{f}(\breve{\mathbf{x}})$, calculated by FEA, to update KS. This iterative process is concluded when the convergence is reached, as shown in Fig. 12.

As depicted in Fig. 11, once the refinement of KS is obtained, i.e., the response surface phase is completed, the optimization step can be performed.

\section{Results}

The obtained Pareto front, reported in Fig. 13, shows two branches, namely $A$ and $C$, characterized by different trends of input variables; in particular, the part $A$ is generated by the set of arrays $\mathbf{x}_{A}$ such that:

$\mathbf{x}_{A}=\left[w_{1}, l, a, b, c\right]^{T}=\left[w_{1}, 2500,0.5-0.6,1.5,1.5\right]^{T}$

with $w_{1}$ varying from $80 \mu \mathrm{m}$ to $200 \mu \mathrm{m}$ for increasing values of COF. Relation (42) states that branch $A$ is only function of the width of the strut $w_{1}$, that may be increased when more scaffolding capabilities are requested. As concerns the length of the strut $l$, the crown radius $b$ (Eq. (18b)) and the width of the link $c$ (Eq. (18c)), their optimal values are obtained increasing them as much as possible, i.e. choosing their maximum allowed in the considered range (see Eq. (35)). Moreover, we observe that the optimal value of the input parameter $a$, that represents the ratio between the width at the center and at the end of the strut (see Eq. (18a)), varies among $0.5-0.6$; consequently, the optimal solutions are obtained when the strut profile is not constant.

The branch $C$ is generated by the the set of arrays $\mathbf{x}_{C}$ such that:

$\mathbf{x}_{C}=\left[w_{1}, l, a, b, c\right]^{T}=[200, l, 0.6-0.64,1.5,1.5]^{T}$

with the length $l$ decreasing from $2500 \mu \mathrm{m}$ to about $1700 \mu \mathrm{m}$ for increasing values of COF. Relation (43) states that branch $C$ is only function of the length of the strut $l$, that may be decreased when 
higher scaffolding is required. Similarly to the case of branch $A$, optimal designs in branch $C$ are obtained by increasing the width of the strut $w_{1}$, the crown radius $b$ and the width of the link $c$ up to their maximum value. The optimal value of the parameter $a$ varies in the range 0.6-0.64.

Better results should be obtained allowing the optimization for higher value of the input variables $l, w_{1}, b$ and $c$ in the design space $\Sigma$. However, such extension will involve the change of the length and of the height of the unit cell, that are fundamental parameters in stent design. The parameter $a$ for the Pareto set shows small oscillations in the reported ranges for branches $A$ and $C$, without a well-defined trend. Accordingly, we considered $a$ nearly constant. It is also important to note that the Pareto optimal set is always represented by tapered strut profiles with a ratio between the width at the center and at the end of the strut ranging in the interval 0.5-0.65.

\section{Discussion}

In order to highlight the improvements in stent fatigue strength achieved by the proposed optimization analysis, we compare the performance of our resulting stent design with the reference de$\operatorname{sign} \mathbf{x}_{R}$.

We note that all the points falling within the area $B R R_{c}$, depicted in Fig. 13, are represented by design parameters that improve, compared to the reference design, the fatigue strength or the scaffolding, or both simultaneously. Moreover, all the points that are on the portion of the Pareto front delimited by points $B$ and $R_{c}$, represent, by definition, the solutions for which it is not possible to further improve the fatigue strength without decreasing scaffolding capabilities and vice-versa. Accordingly, such solutions are equivalent and all of them are optimal designs (in Pareto sense) in the domain $\Sigma$ : the best design trade-off can be selected by restricting the attention to the Pareto set.

As explanatory case study, we show how we maximize fatigue strength, selecting an optimal solution from the Pareto set, under the requirement of leaving COF unchanged with respect to the design $\mathbf{x}_{R}$. Such optimal solution (Fig. 13) is represented by the point $B$ defined as:

$\mathbf{x}_{B}=\left[w_{1}, l, a, b, c\right]^{T}=[163,2493,0.57,1.49,1.4]^{T}$

for which we obtain an alternate strain $\varepsilon_{a}=0.079 \%$ and, consequently, the fatigue safety factor yields:

$S_{B}=\frac{\varepsilon_{L}}{\varepsilon_{a}}=\frac{0.4}{0.079}=5.1$

Comparing Eqs. (38) and (45) we observe that, with the same scaffolding capabilities, introducing the optimized tapered profile (in this case $w_{1}=163 \mu \mathrm{m}, w_{2}=93 \mu \mathrm{m}, l=2493 \mu \mathrm{m}$ ), it is possible to increase the fatigue safety factor under pulsatile loads of approximately 2.4 times.

The effect induced by the introduction of the tapered profile only in the reference design $\mathbf{x}_{R}$ is investigated considering the design resulting from the following input parameters:

$\mathbf{x}_{I}=\left[w_{1}, l, a, b, c\right]^{T}=[120,2000,0.57,1.25,1]^{T}$

The design $\mathbf{x}_{I}$ differs from the reference design $\mathbf{x}_{R}$ (Eq. (36)) only for the value of the parameter $a=0.57$ selected in correspondence with the absolute minimum point for the alternating strain $\varepsilon_{a}$. Therefore, the corresponding geometries are equal, except for the strut profile, which is tapered in the design $\mathbf{x}_{I}$. For such design (Fig. 13) we find an alternating strain $\varepsilon_{a}=0.11 \%$ and a value of the COF equal to $0.19 \mathrm{~N}$. Assuming a fatigue limit of $\varepsilon_{L}=0.4 \%$ the fatigue safety factor reads:

$S_{I}=\frac{\varepsilon_{L}}{\varepsilon_{a}}=\frac{0.4}{0.11}=3.6$
Table 1

Geometrical features and value of the objectives for the typical $\left(\mathbf{x}_{R}\right)$, optimized $\left(\mathbf{x}_{B}\right)$ and tapered $\left(\mathbf{x}_{I}\right)$ design.

\begin{tabular}{llllllll}
\hline Design & $\begin{array}{l}w_{1} \\
(\mu \mathrm{m})\end{array}$ & $\begin{array}{l}l \\
(\mu \mathrm{m})\end{array}$ & $a$ & $b$ & $c$ & $\begin{array}{l}\varepsilon_{a} \\
(\%)\end{array}$ & $\begin{array}{l}\text { COF } \\
(\mathrm{N})\end{array}$ \\
\hline $\mathbf{x}_{R}$ & 120 & 2000 & 1 & 1.25 & 1 & 0.19 & 0.24 \\
$\mathbf{x}_{B}$ & 163 & 2493 & 0.57 & 1.49 & 1.4 & 0.079 & 0.24 \\
$\mathbf{x}_{I}$ & 120 & 2000 & 0.57 & 1.25 & 1 & 0.11 & 0.19 \\
\hline
\end{tabular}

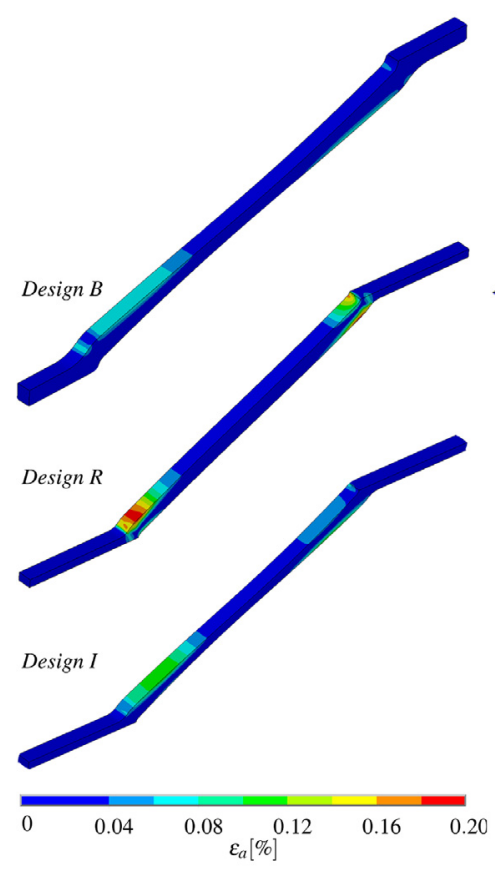

(a)

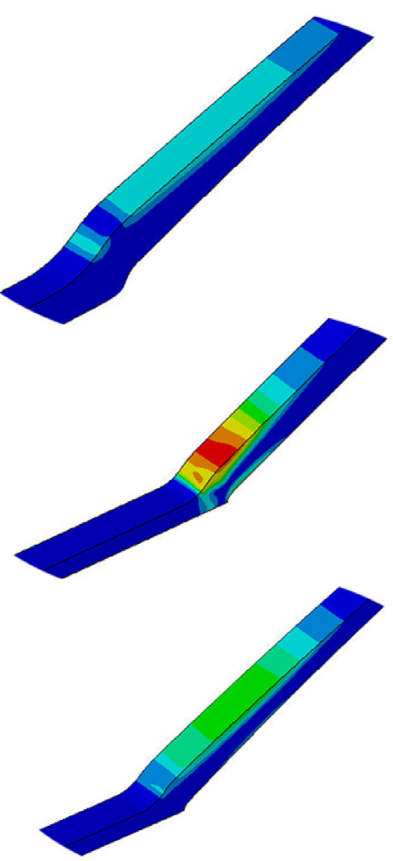

(b)
Fig. 14. (a) Contour plot of the alternating strain $\varepsilon_{a}$ for different designs of the cell: design $B$ represents the optimized profile, design $R$ represents typical $b$ (constant section) profile while design $I$ is equal to $R$ except for the tapered profile. (b) Details of the proposed designs. From top to bottom: design $B, R$ and $I$.

whereas the ratio between the COF corresponding to design points $\mathbf{x}_{I}$ and $\mathbf{x}_{R}$ is $0.19 / 0.24=0.79$. Then, we conclude that only considering the tapered profile allows to increase significantly the fatigue strength but, at the same time, it involves a relative loss of scaffolding capabilities, approximately equal to $20 \%$. Furthermore, as observed as regard to the design $\mathbf{x}_{R}$, the design $\mathbf{x}_{I}$ does not belong to the Pareto set and then it is not a optimal solution in the domain $\Sigma$ : indeed with the same scaffolding of $\mathbf{x}_{I}$ it can be obtained a design with better fatigue strength (point $I_{E}$ in Fig. 13) or, with the same fatigue strength, better scaffolding capabilities may be reached (point $I_{C}$ in Fig. 13). The designs considered in this section are reported in Table 1.

From an analysis of Fig. 14 it is evident that the parts of the cell with higher risk of failure are near to the external part of the crown, as confirmed by other authors [45]. Moreover, the tapered profile allows to redistribute the strain along a wider part of the strut yielding a decrease of its maximum value as discussed in Section 2.4.

In Fig. (15) we show the resulting stent unit cell for both designs $\mathbf{x}_{R}$ and $\mathbf{x}_{B}$. The length and the height of the unit cell are the same for both designs, while the corresponding geometries differ for the strut profile, crown radius and link width. 


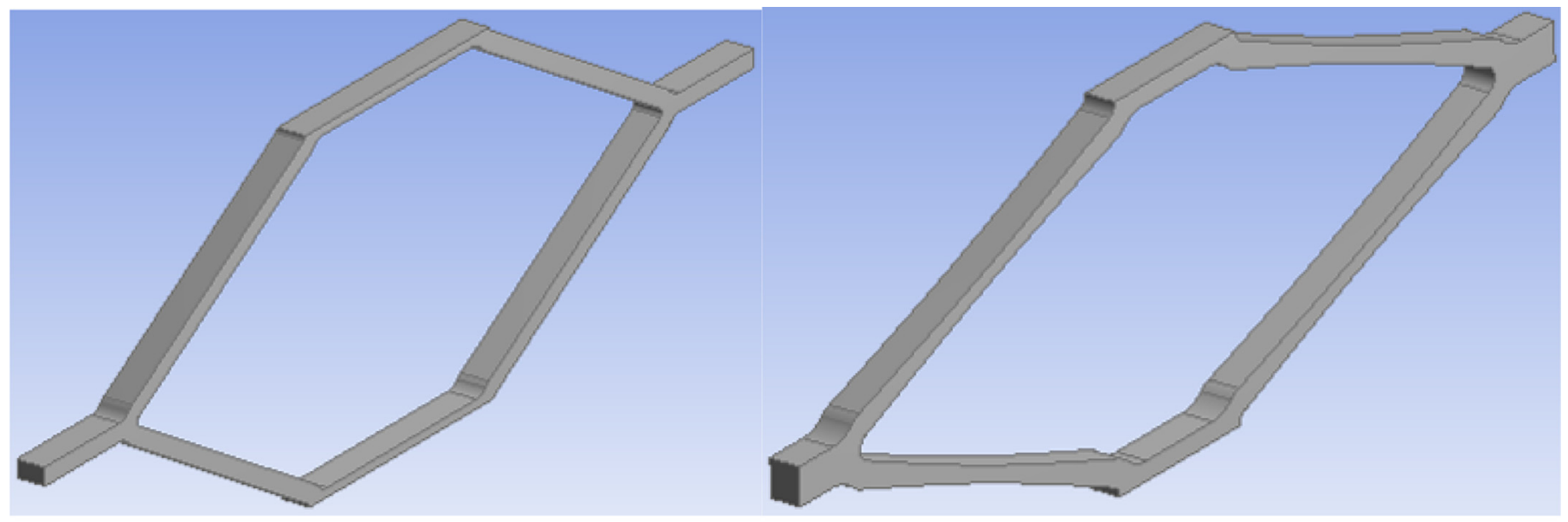

(a)

(b)

Fig. 15. Stent cell geometries. (a) Typical design $R$. (b) Optimized design $B$.

\section{Limitations}

The major limitation of this study lies in the execution of numerical simulations considering the entire cell in a planar configuration. As already highlighted by other authors [19,46,47], it may be considered sufficiently accurate although (i) the actual complete 3D model has curved shape and (ii) stents interact with vessels, resulting in a mechanical response influenced by contact phenomena. Results much closer to the in vivo conditions may be obtained by optimizing complete models of stents. In this regard, it might be useful to carry out experimental tests aimed at validating the proposed optimized design.

It is worth noting that, in addition to the time needed to implement the optimization process (parametric model, boundary and load conditions, post-processing of results), the considered problem requires non-trivial computation times; then, more realistic study require computational platforms such supercomputing.

\section{Conclusions}

The present study discusses the use of multi-objective stent design optimization to enhance fatigue life of self-expanding nitinol stent and vessel scaffolding capability. The results obtained through the proposed optimization study are related to a vessel with assigned compliance and blood pressure variation. The methodology introduced is still valid and can be applied also to different pressure cycles and anatomical positions. The study results confirm that the use of tapered strut profile should be a primary key factor to reduce and uniform the strain field along the strut and thus to enhance the fatigue life of the whole stent. The obtained Pareto set allows the designer for the selection of optimized solution, according to the specific design requirements.

As illustrative example we compared a commercial reference design with an optimized design, chosen from the obtained Pareto set, under the requirement of leaving COF unchanged. The proposed approach suggests that the enhancement of stent fatigue life can be achieved combining tapered strut profile with the following changes in the design of the cell:

- an increase of $25 \%$ of the strut length;

- an increase of $40 \%$ of the strut width at the strut extremities.

Moreover, the results suggest that the width narrowing at the middle of the strut, due to the profile tapering, should be stay among $35-50 \%$.
Under such indications, it is possible to achieve a marked improvement of the fatigue safety factor, i.e., about 2.4 times, compared to the typical design (strut with constant section), without any loss of scaffolding capabilities.

The present study may be used as a starting point for further optimization analyses addressing the design of brand-new peripheral stent models. Further developments can address extension approach to a full 3D case or experimental validation of the achieved results by the performance of fatigue tests for the proposed stent strut design.

\section{Conflict of interest}

None.

\section{Funding}

None.

\section{Ethical approval}

Not required.

\section{Acknowledgments}

Gianluca Alaimo is very grateful to Prof. Mario Di Paola for the precious time he dedicated to the discussion of the themes of the present work and wishes to thank him for his constant support during the writing of the paper.

\section{References}

[1] Auricchio F, Constantinescu A, Conti M, Scalet G. Fatigue of metallic stents: from clinical evidence to computational analysis. Ann Biomed Eng 2016;44:287-301.

[2] Duda S, Bosiers M, Lammer J, Scheinert D, Zeller T, Oliva V, et al. Drug-eluting and bare nitinol stents for the treatment of atherosclerotic lesions in the superficial femoral artery: long-term results from the SIROCCO trial. J Enovasc Ther 2006;13:701-10.

[3] Gibbs JM, Peña CS, Benenati JF. Treating the diseased superficial femoral artery. Tech Vasc Interv Radiol 2010;13(1):37-42.

[4] Carpenter J, Anderson W, Brewster D, Kwolek C, Makaroun M, Martin J, et al. Multicenter pivotal trial results of the lifepath system for endovascular aortic aneurism repair. J Vasc Surg 2004;39:34-43.

[5] Baguet J, Thony F, Sessa C, Mallion J. Stenting of a renal artery compressed by the diaphragm. J Hum Hypertens 2003;17:213-14. 
[6] Knirsh W, Haas NA, Lewin M, Uhlemann F. Longitudinal stent fracture 11 months after implantation in the left pulmonary artery and successful management by a stent-in-stent maneuver. Catheter Cardio Interv 2003;58:116-18

[7] Babalik E, Gulbaran M, Gurmen T. Fracture of popliteal artery stents. Circ J 2003;67:643-5.

[8] Scheinert D, Scheinert S, Sax J, Piorkowski C, Bräunlich S, Ulrich M, et al. Prevalence and clinical impact of stent fractures after femoropopliteal stenting. J Am Coll Cardiol 2005;45(2):312-15.

[9] Allie DE, Hebert CJ, Walker CM. Nitinol stent fractures in the SFA. Endovasc Today 2004;7:22-34

[10] Cheng CP, Wilson N, Hallett R, Herfkens R, Taylor C. In vivo MR angiographic quantification of axial and twisting deformations of the superficial femoral artery resulting from maximum hip and knee flexion. J Vasc Interv Radiol 2006;17:979-87.

[11] Auricchio F, Conti M, Ferraro M, Reali A. Evaluation of carotid stent scaffolding through patient-specific finite element analysis. Int J Numer Methods Biomed Eng 2012;28:1043-55.

[12] Conti M, Van Loo D, Auricchio F, De Beule M, De Santis G, Verhegghe B, et al. Impact of carotid stent cell design on vessel scaffolding: a case study comparing experimental investigation and numerical simulations. J Endovasc Ther 2011:18:397-406.

[13] Petrini L, Migliavacca F, Auricchio F, Dubini G. Numerical investigation of the intravascular coronary stent flexibility. J Biomech 2004;37:495-501.

[14] De Beule M, Van Cauter S, Mortier P, Van Loob D, Van Impec R, Verdoncka P. et al. Virtual optimization of self-expandable braided wire stents. Med Eng Phys 2009;31:448-53.

[15] Grogan JA, Leen SB, McHugh PE. Optimizing the design of a bioabsorbable metal stent using computer simulation methods. Biomaterials 2013;34:8049-60.

[16] Li N, Zhang H, Ouyang H. Shape optimization of coronary artery stent based on a parametric model. Finite Elem Anal Des 2009;45:468-75.

[17] Pant S, Bressloff NW, Limbert G. Geometry parameterization and multidisciplinary constrained optimization of coronary stents. Biomech Model Mechanobiol 2012;11:61-82.

[18] Pant S, Limbert G, Curzen NP, Bressloff N. Multiobjective design optimisation of coronary stents. Biomaterials 2011;32:7755-73.

[19] Wu W, Petrini L, Gastaldi D, Villa T, Vedani M, Lesma E, et al. Finite element shape optimization for biodegradable magnesium alloy stents. Ann Biomed Eng 2010;38(9):2829-40.

[20] Abad EMK, Pasini D, Cecere R. Shape optimization of stress concentration-free lattice for self-expandable nitinol stent-grafts. J Biomech 2012;45:1028-35

[21] Azaouzi M, Lebaal N, Makradi A, Belouettar S. Optimization based simulation of self-expanding nitinol stent. Mater Des 2013;50:917-28.

[22] Duerig TW, Tolomeo DE, Wholey M. An overview of superelastic stent design. Minim Invasive Ther Allied Technol 2000:9(3):235-46.

[23] Pelton AR, Schroeder V, Mitchell MR, Gong XY, Barney M, Robertson SW. Fatigue and durability of nitinol stents. J Mech Behav Biomed 2008;1:153-64.

[24] Duerig TW, Burpee J, Mathis M. Intravascular stent having tapered struts. Tech. Rep., U.S. Patent Number 6190406; 2001.

[25] Hsiao HM, Yin MT. An intriguing design concept to enhance the pulsatile fatigue life of self-expanding stents. Biomed Microdevices 2014;16:133-41.

[26] Deb K, Pratap A, Agarwal S, Meyarivan T. A fast and elitist multiobjective genetic algorithm: NSGA-II. IEEE Trans Evol Comput 2002;6(2):182-97.
[27] Robertson SW, Pelton AR, Ritchie RO. Mechanical fatigue and fracture of nitinol. Int Mater Rev 2012;57(1):1-37.

[28] Pelton AR. Nitinol fatigue: a review of microstructures and mechanisms. J Mater Eng Perform 2011;20:613-17.

[29] Anderson TL. Fracture mechanics: fundamentals and applications. 3rd ed. CRC Press; 2005.

[30] Robertson SW, Ritchie RO. A fracture-mechanics-based approach to fracture control in biomedical devices manufactured from superelastic nitinol tube. J Biomed Mater Res B 2008;84:26-33.

[31] Stoeckel D, Bonsignore C, Duda S. A survey of stent designs. Minim Invasive Ther Allied Technol 2002;11(4):137-47.

[32] Myers RH, Montgomery DC, Anderson-Cook CM. Response surface methodology: process and product optimization using designed experiments. 4th ed. Wiley; 2016.

[33] Romano F. Deflections of Timoshenko beam with varying cross-section. Int J Mech Sci 1996;38:1017-35.

[34] Balduzzi G, Aminbaghai M, Sacco E, Füssl J, Eberhardsteiner J, Auricchio F. Non-prismatic beams: a simple and effective Timoshenko-like model. Int J Solids Struct 2016;90:236-50.

[35] Paglietti A, Carta G. Remarks on the current theory of shear strength of variable depth beams. TOCIEJ 2009;3:28-33.

[36] Auricchio F, Taylor RL, Lubliner J. Shape-memory alloys: macromodeling and numerical simulations of the superelastic behavior. Comput Methods Appl Mech Eng 1997;146:281-312.

[37] Meoli A, Dordoni E, Petrini L, Migliavacca F, Dubini F, Pennati G. Computational modelling of in vitro set-ups for peripheral self-expanding stents: the importance of stent-wall interaction in the assessment of the fatigue resistance. Cardiovasc Eng Technol 2013:4:474-84.

[38] Benetos A, Laurent S, Hoeks AP, Boutouyrie PH, Safar ME. Arterial alterations with aging and high blood pressure. A non-invasive study of carotid and femoral arteries. Arterioscler Thromb Vasc Biol 1993;13:90-7.

[39] Kawasaki T, Sasayama S, Yagi SI, Asakawa T, Hirai T. Non-invasive assessment of the age related changes in stiffness of major branches of the human arteries. Cardiovasc Res 1987;21(9):678-87.

[40] Kaymaz I. Application of Kriging method to structural reliability problems. Struct Saf 2005;27:133-51.

[41] Sacks B, Miller A, Gottlieb M. Fracture of an iliac artery Palmaz stent. J Vasc Interv Radiol 1996;7:53-5.

[42] Sacks J, Welch WJ, Mitchell TJ, Wynn HP. Design and analysis of computer experiments. Stat Sci 1989;4(4):409-23.

[43] https://www.cordis.com/global-home.html [accessed: 17.01.17]. 2017.

[44] SAS-IP. Ansys design exploration. 12th ed. Ansys Inc.; 2009.

[45] Gong XY, Pelton AR, Duerig TW, Rebelo N, Perry K. Finite element analysis and experimental evaluation of superelastic nitinol stent. In: SMST 2003 - international conference on shape memory and superelastic technologies; 2003. p. 443-51.

[46] Donnelly EW, Bruzzi MS, Connolley T, McHugh PE. Finite element comparison of performance related characteristics of balloon expandable stents. Comput Methods Biomech Biomed Eng 2007;10:103-7.

[47] Mori K, Saito T. Effects of stent structure on stent flexibility measurements. Ann Biomed Eng 2005;33:733-42. 